\title{
Microwave Assisted Ignition to Achieve Combustion Synthesis ${ }^{\dagger}$
}

\author{
E. BALAKRISHNAN* \\ balak@squ.edu.om \\ Department of Mathematics and Statistics, Sultan Qaboos University, Sultanate of \\ Oman.
}

M.I. NELSON ${ }^{* *}$

mark@ma.adfa.edu.au

School of Mathematics and Statistics, University College, UNSW, Canberra ACT 2600, AUSTRALIA

X.D. CHEN

d.chen@auckland.ac.nz

Department of Chemical \& Materials Engineering, University of Auckland, New Zealand.

\begin{abstract}
The use of microwave heating to initiate combustion synthesis has been increasingly investigated in recent years because of its advantages over traditional methods. A simple mathematical model is used to model these experiments. The microwave power absorption term is modelled as the product of an Arrhenius reaction term with a function that decays exponentially with distance. The former represents the temperaturedependent absorption of the microwaves whereas the latter describes the penetration of the material by the microwaves. Combustion kinetics are modelled as a first-order Arrhenius reaction.
\end{abstract}

Keywords: ceramics, combustion, microwaves, self-propagating high-temperature synthesis (SHS).

\section{Introduction}

Microwave heating is fundamentally different from conventional heating in that heat is generated internally material-microwave interaction (absorption), rather than originating on the exterior surface of the sample. This heating produces thermal gradients that are the reverse of those observed under conventional heating. Furthermore the heating can be both rapid and selective. Consequently, microwave processing makes it possible to

$\dagger$ Due to space limitation this article was omitted from the previous special issue on McNabb Symposium.

* Requests for reprints should be sent to E. Balakrishnan, Department of Mathematics and Statistics, Sultan Qaboos University, Sultanate of Oman.

** Parts of this paper were completed while the author was at the Department of Fuel and Energy, The University of Leeds, Leeds LS2 9JT, UK. 
heat bodies of various shapes and sizes very rapidly and relatively uniformly.

Microwave heating has been used in the food industry since the early 1960s. A wide range of applications in materials processing are currently being investigated[4], [14]: recovery, synthesis, removal of phases, fabrication, consolidation, and post-fabrication treatments. These processes offer the prospect of significant reductions in manufacturing costs due to energy savings and short processing times.

Inorganic materials that are thermodynamically stable at high temperatures, e.g. ceramics, have numerous technological applications, e.g. high temperature electronics, high temperature furnaces, structural materials in aircraft engines etc. The conventional manufacture and processing of these materials often involve processes that are extremely tedious, involving many cumbersome steps, and incorporating combinations of high temperatures, long reaction times (e.g. synthesis of many metal chalcogenides require a week or more to complete), and specialised apparatus. Such methods include: the direct reaction of fused high purity constituents; reaction of arc melted pure metals; laser pulse treatment at high pressures; rigorous mechanical alloying; plasma heating; combustion synthesis, etc.

In combustion synthesis, also known as self-propagating high-temperature synthesis (SHS), the product is formed via a sufficiently exothermic reaction by initiating a self-sustaining reaction wave that propagates through the reactants[11]. An example of combustion synthesis is the formation of silicon carbide:

$$
\mathrm{Si}+\mathrm{C} \rightarrow \mathrm{SiC} ; \quad \Delta \mathrm{H}_{298}^{o}=-66.9 \mathrm{kJmol}^{-1}[10] .
$$

Microwave heating has been used recently to accelerate solid state reactions. Its advantages over conventional methods include very short time scales for the preparation of the reactants, increases in reaction rates, a substantial reduction in processing times, higher yields, lower operating temperatures, the simplicity of the method, selectivity in energy transfer from the microwave field, improved structural uniformity in the product, improved or unique microstructures and properties, and synthesis of new materials. Combustion synthesis can be ignited by microwave energy internally and forced to propagate outward in a controllable manner[3], in contrast with self-propagation in conventional combustion synthesis. At present certain products can only be fabricated or synthesised by microwave heating or by a combination of microwave and conventional heating.

Microwave heating is particularly effective for highly exothermic processes because absorption increases with temperature; at higher temperatures there is a higher dissipation of energy and a corresponding increase 
in temperature. Thermal runaway can occur in chemically inert materials, entirely due to positive feedback from thermal absorption[14]. The synergy between combustion synthesis and microwave heating further escalates the reaction rate. Thermal runaway has been observed in mixtures of $\mathrm{Cu}_{2} \mathrm{O}+\mathrm{C}, \mathrm{Ag}_{2} \mathrm{O}+\mathrm{C}$, and $\mathrm{V}_{2} \mathrm{O}_{5}+\mathrm{ZrO}_{2}$ heated by microwaves[6].

Recent applications of microwave heating includes synthesis of $\beta$-SiC powder[13], preparation of metal vanadates and chalcogenides[15], microwavehydrothermal processing of metal powders[9], formation of metals and carbides under atmospheric pressure[6], and the preparation of transition metal silicides[16].

In this paper we use a simple model to illustrate the initiation of combustion synthesis by microwave heating.

\section{Description of the model}

We assume that the sample is a 1-dimensional slab. On the boundaries $x=$ 0 and $x=2 L$ the slab undergoes convective and radiative heat-transfer with its surroundings. Both sides of the slab are exposed to a microwave field so that the intensity of the radiation is the same at $x=0$ and $x=L$. This last condition is usually achieved experimentally by rotating the sample. We therefore impose a symmetry condition at $x=L$, equation (5). The material undergoes an exothermic reaction which is described by first-order Arrhenius kinetics.

\subsection{Mathematical modelling of microwave heating of a solid material}

The internal temperature profile of a material that is heated by microwaves is governed by a reaction-diffusion equation in which the source term due to microwave heating is given by

$$
\mathcal{R}=q(T)\left\|E_{i}\right\|^{2},
$$

where the function $q(T)$ models the thermal absorption of microwave energy by the sample and $\left\|E_{i}\right\|^{2}$, the square of the electric-field amplitude, represents the penetration of the microwaves into the interior of the material. The latter quantity is defined by Maxwell's equations, which govern the propagation of electromagnetic radiation in a dielectric medium. In the most general case the dielectric properties of a medium are temperature dependent and so the equations for the spatial variation of the electric and magnetic fields are coupled to a nonlinear heat-conduction equation. The 
solution of such a system is a formidable task. Brevity prevents a discussion of recently developed models of these complex physical processes. The interested reader is referred to a review article[8].

In order to present a simplified account of microwave heating we assume that the electric field amplitude decays exponentially with distance (the Beer Lambert Law). This represents the simplest possible spatial dependence which has some accepted physical basis but which ennables the heating aspects of the problem to be isolated from the electric and magnetic fields. The use of the Beer-Lambert law in simple microwave heating models is discussed elsewhere[12]. The conditions under which the BeerLambert Law provides a good approximation to the solutions of Maxwell's equations have been investigated by Ayappa[1].

We assume that thermal absorption function is given by an exponential function which is normalised to have unitary value at room temperature,

$$
q(T)=\exp \left[T_{m}\left(\frac{1}{T_{r}}-\frac{1}{T}\right)\right] .
$$

\section{Model equations}

\subsection{Dimensionalised model}

The system we study is

$$
\begin{aligned}
k \frac{\partial^{2} T}{\partial x^{2}}= & \begin{array}{l}
\text { Reaction-Diffusion Equation } \\
\rho c
\end{array} \quad Q \rho \frac{\partial T E}{R T_{c}^{2}} \exp \left[\frac{E}{R}\left(\frac{1}{T_{c}}-\frac{1}{T_{s}}\right)\right] \\
& -\alpha_{m} Q_{m} \exp [-\kappa x] \exp \left[T_{m}\left(\frac{1}{T_{r}}-\frac{1}{T}\right)\right], \\
& \text { Boundary Condition on } x=0
\end{aligned}
$$

In equation (3) the kinetics have been in terms of a characteristic temperature and an activation energy, rather than the standard Arrhenius formulation. The advantages of this formulation are outlined elsewhere[2]. 
Note that in equation (4) $\alpha$ is an averaged emissivity over all the available spectra, whilst in equation (3) $\alpha_{m}$ is specific to the microwave wavelength. In what follows we treat the product $\alpha_{m} Q_{m}$ as a bifurcation parameter since in practice this is what is measured.

\subsection{Non-dimensionalised model}

In non-dimensionalising equations (3-6) we introduce a non-dimensionalised length, $x^{*}$, a non-dimensionalised temperature, $T^{*}$, and a non-dimensionalised time, $t^{*}$. These are defined in the nomenclature. Our system can then be written as

$$
\begin{aligned}
& \text { Reaction-Diffusion Equation } \\
\frac{\partial^{2} T^{*}}{\partial x^{*^{2}}}= & \frac{\partial T^{*}}{\partial t^{*}}-\frac{\Phi L^{*^{2}} E^{*}}{k^{*} T_{c}^{*^{2}}} \exp \left[E^{*}\left(\frac{1}{T_{c}^{*}}-\frac{1}{T^{*}}\right)\right] \\
& -\frac{\Phi_{m} L^{*^{2}}}{k^{*}} \exp \left[-\kappa^{*} L^{*} x^{*}\right] \exp \left[T_{m}^{*}\left(1-\frac{1}{T^{*}}\right)\right], \\
& \text { Boundary condition on } x^{*}=0 . \\
-\frac{\partial T^{*}}{\partial x^{*}}= & \frac{L^{*}}{k^{*}} \cdot\left\{\left(T_{a}^{*}-T^{*}\right)+\alpha q_{\text {out }}\left(T_{a}^{*^{4}}-T^{*^{4}}\right)\right\}, \\
-\frac{\partial T^{*}}{\partial x^{*}}= & 0 \\
& \text { Boundary condition on } x^{*}=1 . \\
T^{*}\left(x^{*}, 0\right)= & T_{a}^{*},
\end{aligned}
$$

The non-dimensionalisation has been chosen so that each of the parameters of interest $\left(T_{a}, L, \alpha_{m} Q_{m}, \kappa, T_{m}\right.$ etc) appears in only one nondimensionalised variable $\left(T_{a}^{*}, L^{*}, \Phi_{m}^{*}, \kappa^{*}, T_{m}^{*}\right.$ respectively). Hence in what follows we often refer to the critical ambient temperature rather than the critical non-dimensionalised ambient temperature, etc.

The notation is a little awkward since on the one hand the numerical package used requires that the equations be solved on $[0,1]$, requiring the introduction of a length-scale $x^{*}=x / L$, whereas for interpretation of the results the sample length should not appear in any of the other control parameters. 


\subsection{Numerics}

The steady-state solutions of equation (7) with boundary conditions (8)-(9) was investigated using Auto 97[5] by rewriting the initial-value problem as a boundary value problem.

In the steady-diagrams, figure $1 \& 2$ (a), we do not show the solution branch corresponding to solutions when the continuation parameters, $T_{a}^{*}$ and $\Phi_{m}^{*}$ respectively, are negative. The default parameter values used in the numerical calculations are stated following the Nomenclature.

\section{Results}

A standard method to initiate combustion synthesis is to place the reactant in an hot atmosphere, i.e. to use ambient temperature as the continuation parameter. An overview of this classic combustion problem is provided elsewhere[2]. Figure 1 shows partial steady-state diagrams for this scenario. Solution (a) represents a laboratory investigation using a small sample $(L=0.05 \mathrm{~m})$ and solution (b) a scaled-up sample for industrial use $(L=0.50 \mathrm{~m})$. Preliminary laboratory investigations may use even smaller samples. However, if the thickness is sufficiently small the microwave intensity does not decrease exponentially within the sample and must be found by solving Maxwell's equations[1]. Thus the assumption that the decay is exponentially imposes a restriction on the minimal sample size as used in this study.

Both steady-state curves contain two branches and one limit point. The lower-branch, the no-ignition branch, represents stable solutions in which the maximum temperature is slightly higher than ambient. These solutions are states of negligible reaction. The second branch corresponds to unstable solutions. In addition there is a high-valued branch of stable solutions, not shown on the diagram, corresponding to an ignited state. This combustion branch represents combustion synthesis. When ambient temperature is used as the continuation parameter the criticality condition for combustion synthesis is given by the limit point at which the lower solution branch is terminated, we refer to it as the ignition limit point. The ignition temperatures are $T_{a, c r}=963.2 \mathrm{~K}$, when $L=0.50 \mathrm{~m}$, and $T_{a, c r}=638.4 \mathrm{~K}$, when $L=0.05 \mathrm{~m}$. As the sample size decreases the critical temperature required to initiate the reaction increases dramatically. Thus it is desirable to investigate other mechanisms for initiating combustion synthesis.

Due to the structure of the steady-state diagrams in figure 1 the combustion branch exists at room temperature $\left(T_{a}^{*}=1\right)$. Thus once combustion 


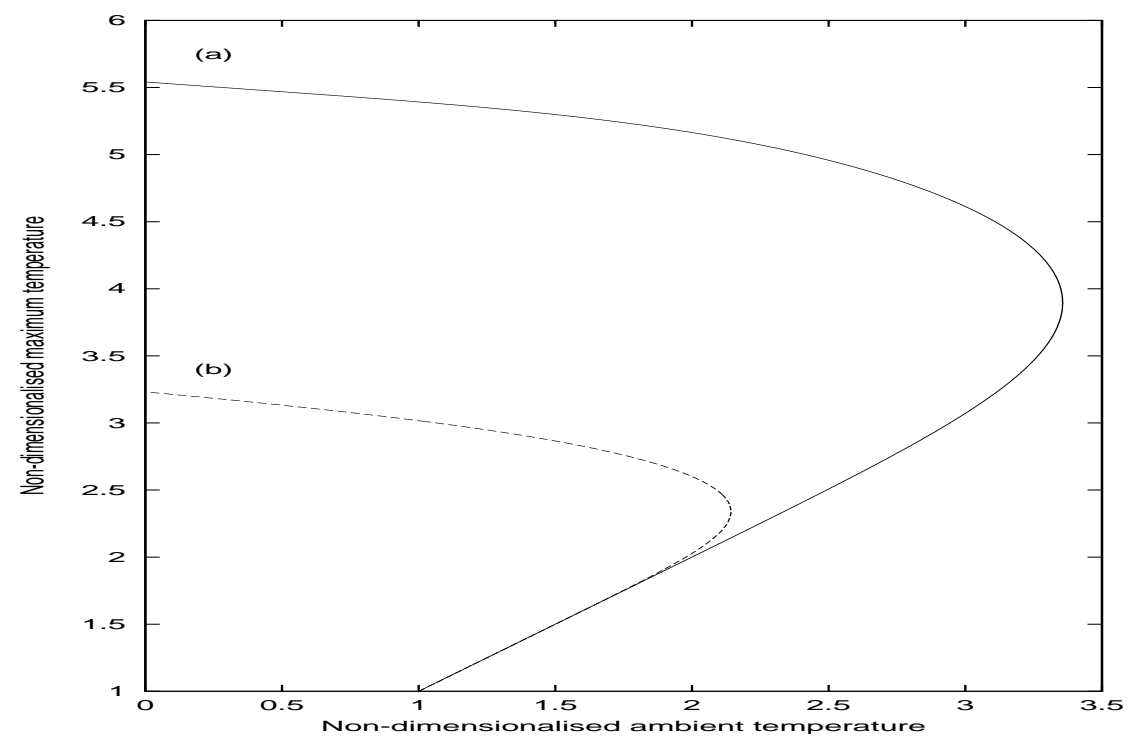

Figure 1. Steady-state diagram when microwave heating is not used: the effect of sample size. Note that the ignition-branch is not shown. Parameter values: sample half-length length, (a) $L=0.05 \mathrm{~m}$ (b) $L=0.50 \mathrm{~m}$.

synthesis has been initiated the microwave power source can be turned off as the reaction is self-propagating.

Figure 2 (a) shows the steady-state diagram when microwave heating is used. Both curves contain three limit points, defining four solution branches. The lowest branch again represents a stable state of negligible chemical reaction. No comment can be made about the stability of the other branches in figure 2 (a) as Auto does not calculate solution stability in boundary-value problems. The highest combustion branch is not shown. As the microwave number is increased the no-ignition branch is terminated at a limit point and this therefore defines the minimum power needed to initiate combustion synthesis. Note that as the combustion branch exists when the the power has been turned off $\left(\Phi_{m}^{*}=0\right)$ combustion synthesis may be initiated by a finite pulse of microwave energy. Herein we do not address the question of the required pulse duration.

Figure 2 (a) shows that increasing the sample size by a factor of ten has negligible effect on the criticality condition. In figure 2 (b) the ignition limit point is unfolded with the sample half-length for three values of the decayconstant. At the lowest value, curve (1), there is a very slight variation in 


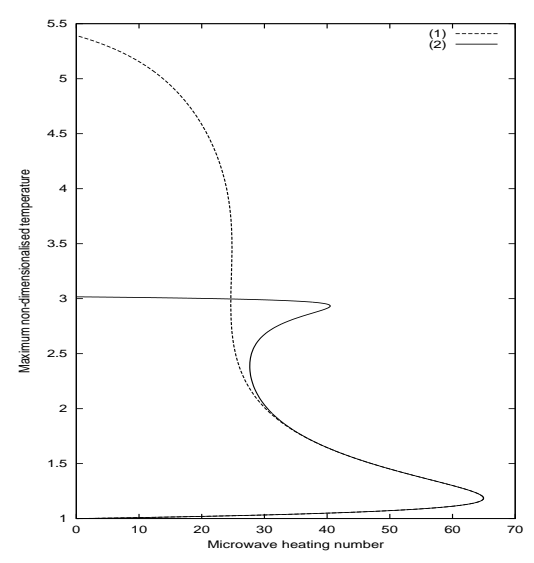

(a) Steady-state diagram when microwave heating is used.

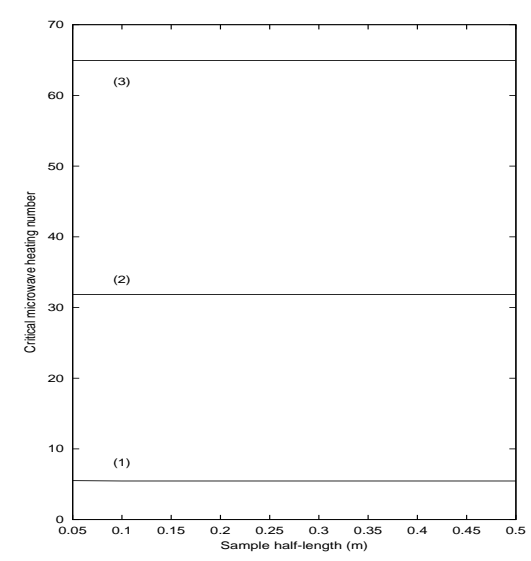

(b) Variation of the critical nondimensionalised microwave heating number $\left(\Phi_{m, c r}^{*}\right)$ with the sample half-length $L(\mathrm{~m})$.

Figure 2. Parameter values. Figure (a): Sample half-length; (1) $L=0.05 \mathrm{~m}$, (2) $L=$ $0.50 \mathrm{~m}$. Figure (b): Decay constant; (1) $\kappa^{*}=10^{2},(2) \kappa^{*}=5 \times 10^{2},(3) \kappa^{*}=10^{3}$.

the critical microwave number at small values of the half-length, but not enough to show on the scale of the figure. In the remaining curves there is no meaningful variation.

In figure 3 the ignition limit point is unfolded with the the decay constant $(\kappa)$ and the activation temperature for thermal absorption $\left(T_{m}\right)$. Although for a given material form these are fixed their value can be controlled by the addition of chemically inert coupling/absorbing agents (e.g. SiC, carbon, binders) to the reactants. The coupling agent interacts with the microwave field and acts as an in situ inert heating source; pre-heating the sample, i.e. changing the value for $\kappa$ and/or $T_{m}$. Other methods that may be used to increase absorption include[4]: the alteration of the microstructure and defect structure of the sample, by changing their form (e.g. bulk vs powder), and by changing the frequency of the incident radiation. Such methods are used when none of the reactants is a good microwave susceptor.

This figure shows that the critical microwave number increases linearly with the decay constant and decreases with increasing activation temperature. As the decay constant increases adsorption of the microwave energy is increasingly concentrated into a narrow boundary-layer near the sample 


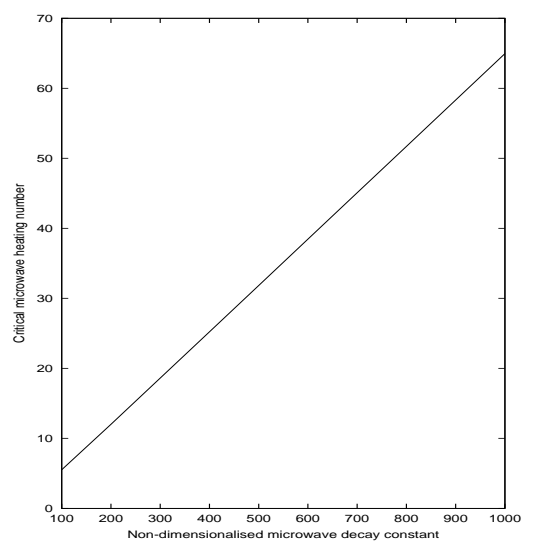

(a)

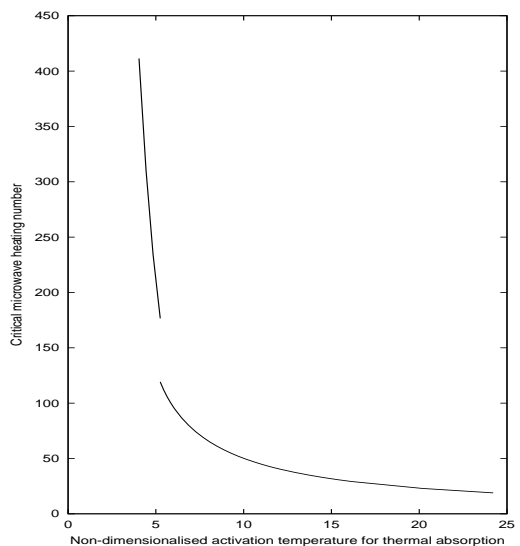

(b)

Figure 3. Variation of the critical microwave heating number $\left(\Phi_{m, c r}^{*}\right)$ with: (a) the non-dimensionalised decay constant $\left(\kappa^{*}\right)$; (b) the non-dimensionalised activation temperature for thermal absorption $\left(T_{m}^{*}\right)$.

surface. Under these circumstances heat-loss on the boundary $x^{*}=0$ becomes more important in determining the net energy absorbed. An increase in the decay constant is therefore expected to increase the criticality condition. The microwave adsorption term, equation (2), is an increasing function of the activation temperature provided that the temperature is greater than ambient. Increasing the activation temperature therefore increases energy dissipation, decreasing the critical microwave number. However, the decrease is not continuous: a small increase in the activation temperature above the value $T_{m}^{*} \approx 5.247$ reduces the critical microwave number by almost one-third.

This dramatic decrease is explained by a change in the steady-state structure. Figure 4 shows that when $T_{m}^{*}=5.247$ there is only one limit point (L1') in the positive quadrant, with value $\Phi_{m, c r}^{*}=176.6$. For a slightly higher value of the activation temperature, $T_{m}^{*}=5.328$, there are three limit points in the right-hand quadrant with values: 166.9 (L3), 116.1 (L2) and $\Phi_{m, c r}^{*}=115.6(\mathrm{~L} 1)$. Thus in the range $5.247<T_{m}^{*}<5.328$ there is a quartic-fold bifurcation[7]. This bifurcation is responsible for the dramatic non-continuous change in the ignition limit-point. 


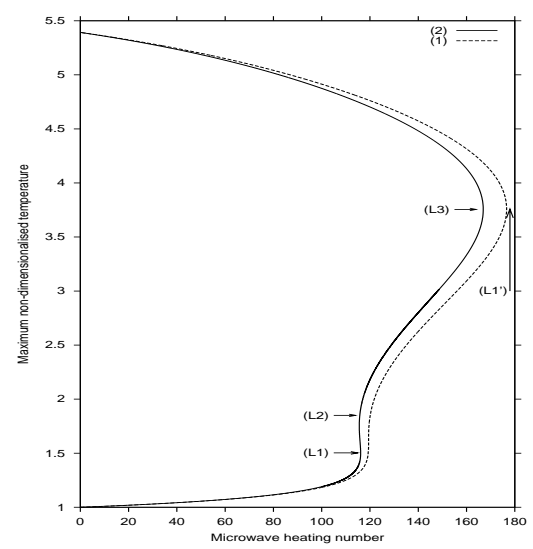

(a)

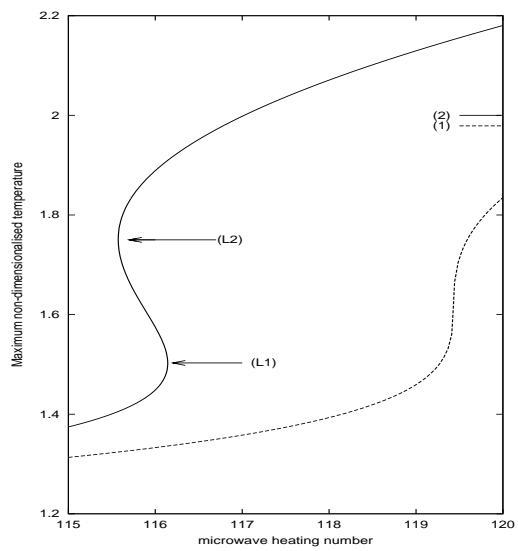

(b) Blow up of figure (a) near the limit points (L1) \& (L2).

Figure 4. Variation of maximum non-dimensionalised temperature with microwave heating number $\left(\Phi_{m}^{*}\right)$. Parameter values: non-dimensionalised activation temperature for thermal absorption; (1) $T_{m}^{*}=5.247$, (2) $T_{m}^{*}=5.328$.

The structure of the steady-state curve following the quartic-fold bifurcation question raises an intriguing point. In the range $119<\Phi_{m}^{*}<176.6$ the maximum temperature on the no-ignition branch for curve (1) is comparatively high: at criticality it is approximately 3.76. In curve (2) the solutions between the (L1) and (L2) fold points are unstable. However, it is possible that the comparatively high-valued solutions between the (L2) and (L3) fold points are stable - they are close in value to the corresponding solutions on curve (1). If this happens then combustion synthesis is not necessarily initiated by increasing the microwave number past (L1): the solution could jump to the branch between (L2) and (L3). (Even if this branch is stable the solution will only evolve onto it if the initial condition is in its basin of attraction, it might be in the basin of attraction for the combustion branch). Under these circumstances thermal runaway would only occur when the microwave heating number is increased past (L3). In this scenario the critical microwave number is a continuous function of the activation temperature and the branch between (L2) and (L3) could phenomenalogically be described as 'smouldering-type behaviour'.

A comparison of figures 2 (a) \& 4 reveals that as the activation temperature is increased over the range $5.328<T_{m}^{*}<8.072$ there is a double 
limit point bifurcation[7]. The practical consequence of this is that the (L3) limit point is now the left of the ignition limit point. Thus although 'smouldering states' still exist, increasing the microwave number past (L1) initiates combustion synthesis.

We intend addressing these issues at a latter date.

\section{Conclusion}

We have investigated some phenomena revealed by a simple model for the microwave initiation of combustion synthesis in which the intensity of the irradiation decays exponentially with distance inside the sample (the BeerLambert Law). There is a critical value of the microwave heating number $\left(\Phi_{m, c r}^{*}\right)$, corresponding to a limit-point bifurcation, at which the no-ignition branch loses stability. As the microwave number is increased past criticality two scenarios are envisaged: (1) the only solution branch is the combustion branch, in which case combustion synthesis is initiated; (2) there are two stable solution branches, an ignition branch and a 'smouldering' branch. In the latter case the solution may evolve onto the middle branch. The stability of the middle branch is lost at a third limit point, increasing the microwave number past this value initiates combustion synthesis. At present the stability of the 'smouldering' state is conjectured.

The ignition limit point has been unfolded with the sample half-length $(L)$, the microwave decay constant $(\kappa)$ and the activation temperature $\left(T_{m}\right)$. Each of these parameters may be manipulated in practice. Over the range of values used the critical microwave number was, for all practical purposes, independent of sample length and increased linearly with the decay constant. Due to the presence of a quartic fold bifurcation, and subject to the possibility of 'smouldering' behaviour, there is a discontinuous decrease in the critical microwave number as the activation temperature is decreased. Thus the addition of a small amount of additive which is more absorptive to microwaves is expected to dramatically decrease the power level required to initiate combustion synthesis.

\section{Acknowledgments}

MIN and EB thank Alex McNabb for his support and encouragement whilst they were at the University of Auckland. We greatly appreciated his insights and comments on the interpretation of the mathematics of nonlinear partial differential equations in terms of the 'real-world' behaviour of the systems that we were modeling. 
This work was carried out whilst MIN was supported by the EPSRC (GR/L28142).

\section{References}

1. K.G. Ayappa, H.T. Davis, G. Crapiste, E.A. Davis, and J. Gordon. Microwave Heating: An Evaluation of Power Formulations. Chemical Engineering Science, 46(4):1005-1016, 1991.

2. E. Balakrishnan, M.I. Nelson, and G.C. Wake. Radiative ignition of combustible materials I. Polymeric materials undergoing non-flaming thermal degradation The critical storage problem. Mathematical and Computer Modelling, 30:177-195, 1999.

3. D.E. Clark, I. Ahmad, and R.C. Dalton. Microwave ignition and combustion synthesis of composites. Materials Science and Engineering A, 144:91-97, 1991.

4. D.E. Clark and W.H. Sutton. Microwave Processing of Materials. Annual Review of Materials Science, 26:299-331, 1996.

5. E.J. Doedel, T.F. Fairgrieve, B. Sandstede, A.R. Champneys, Y.A. Kuznetsov, and X. Wang. AUTO 97: Continuation and bifurcation software for Ordinary Differential Equations (with HomCont), March 1998. Available by anonymous ftp from ftp.cs.concordia.ca/pub/doedel/auto.

6. M. Gasgnier, A. Petit, H. Jullien, and A. Loupy. Microwave-monomode energy transfer: Chemical syntheses, crystallographric and thermal properties of mineral powders. Materials Research Bulletin, 11(9):1101-1109, 1996.

7. B.F. Gray and M.J. Roberts. A method for the complete qualitative analysis of two coupled ordinary differential equations dependent on three parameters. Proceedings of the Royal Society A, 416:361-389, 1988.

8. J.M. Hill and T.R. Marchant. Modelling microwave heating. Applied Mathematics and Modelling, 20:3-15, 1996.

9. S. Komarneni, R. Pidugu, Q.H. Li, and R. Roy. Microwave-hydrothermal processing of metal powders. Journal of Materials Research, 10(7):1687-1692, July 1995.

10. O. Kubaschewski and C.B. Alcock. Metallurgical Thermochemistry. Pergamon Press, Oxford, England, 1989.

11. A.G. Merzhanov. Fundmanetals, achievements, and perspectives for development of solid-flame combustion. Russian Chemical Bulletin, 46(1):1-27, January 1997.

12. M.I. Nelson, G.C. Wake, X.D. Chen, and E. Balakrishnan. The multiplicity of steady-state solutions arising from microwave heating: I Infinite Biot number and small penetration depth. Accepted for publication in Journal of the Australian Mathematical Society B, April 1999.

13. P.D. Ramesh, B. Vaidhyanathan, M. Ganguli, and K.J. Rao. Synthesis of $\beta-\mathrm{SiC}$ powder by use of microwave radiation. Journal of Materials Research, 9(12):30253027, December 1994.

14. W.H. Sutton. Microwave Processing of Ceramic Materials. American Ceramic Society Bulletin, 68(2):376-386, 1989.

15. B. Vaidhyanathan, M. Ganguli, and K.J. Rao. Fast solid state synthesis of metal vanadates and chalcogenides using microwave irradiation. Materials Research Bulletin, 30(9):1173-1177, 1995.

16. B. Vaidhyanathan and K.J. Rao. Microwave assisted synthesis of technologically important transition metal silicides. Journal of Materials Research, 12(12):32253229, 1997. 


\section{Appendix}

\section{Nomenclature}

The subscript $\mathrm{cr}$ is the value that a parameter takes at criticality.

E Activation energy.

$E^{*} \quad$ Non-dimensionalised activation energy. $E^{*}=\frac{E}{R T_{r}}$

$\mathcal{H}$ Ramping rate used in a thermogravimetric experiment. $\left(\mathrm{Ks}^{-1}\right)$

$L \quad$ The half-length of the test sample

$L^{*} \quad$ Non-dimensionalised sample half-length. $L^{*}=L / L_{r}$

$L_{r} \quad$ A reference length-scale.

$Q \quad$ Reaction exothermicity.

$Q_{m} \quad$ Rate of heat generation due to microwaves.

$R \quad$ The ideal gas constant.

$T$ Temperature.

$T^{*} \quad$ Reduced temperature scale. $T^{*}=T / T_{r}$

$T_{a} \quad$ Ambient temperature.

$T_{c} \quad$ Characteristic temperature measured in a $(\mathrm{K})$. thermogravimetric experiment.

$(\mathrm{K})$.

$T_{m} \quad$ The activation temperature for thermal absorption.

$T_{j}^{*} \quad$ A non-dimensionalised temperature $(\mathrm{j}=\mathrm{a}, \mathrm{c}, \mathrm{m})$. $T_{j}^{*}=T_{j} / T_{r}$

$T_{r} \quad$ Reference temperature.

$(\mathrm{K})$

c Heat capacity.

$k \quad$ Thermal conductivity.

$k^{*} \quad$ Non-dimensionalised thermal conductivity. $k^{*}=\frac{k}{\chi_{1} L_{r}}$

$q_{\text {out }}$ Non-dimensionalised radiative heat loss coefficient.

$q_{\text {out }}=\frac{\sigma T_{r}^{3}}{\chi_{1}}$

$t \quad$ Time.

$t^{*} \quad$ Non-dimensionalised time.

$t^{*}=\left(\frac{k}{\rho c L^{2}}\right) t$

$x \quad$ Position within the test sample.

$0 \leq x \leq L$

$x^{*} \quad$ Reduced length scale.

$x^{*}=x / L$ 
$\Phi \quad$ Non-dimensionalised reaction exothermicity.

$\Phi=\frac{Q \rho H L_{r}}{\chi_{1} T_{r}^{2}}$

$\Phi_{m} \quad$ Microwave heating number.

$\Phi_{m}=\alpha_{m} \cdot \frac{Q_{m} L_{r}}{T_{r} \chi_{1}}$

$\alpha \quad$ Absorptivity.

$0 \leq \alpha \leq 1$

$\alpha_{m}$ The absorptivity of the material at the wavelength of the microwaves.

$\rho \quad$ Density.

$\sigma \quad$ Stefan-Boltzmann constant.

$\chi_{1} \quad$ The heat transfer coefficient between the sample and the surrounding air.

$\kappa \quad$ Decay constant for microwave heating.

$\kappa^{*} \quad$ Non-dimensionalised decay constant for microwave heating. $\kappa^{*}=\kappa \cdot L_{r}$.

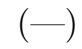

$\left(\mathrm{kg} \mathrm{m}^{-3}\right)$

$\left(\mathrm{Wm}^{-2} \mathrm{~K}^{-4}\right)$

$\left(\mathrm{Wm}^{-2} \mathrm{~K}^{-1}\right)$ $\left(\mathrm{m}^{-1}\right)$

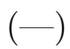

Unless otherwise specified we take the following typical parameter values: $E=80 \times 10^{3} \mathrm{Jmol}^{-1}, \mathcal{H}=1 / 60 \mathrm{Ks}^{-1}, L=0.05 \mathrm{~m}, L_{r}=1.00 \mathrm{~m}$, $Q=3 \times 10^{6} \mathrm{Jkg}, T_{a}=298 \mathrm{~K}, T_{c}=1380 \mathrm{~K}, T_{m}=2405.46 \mathrm{~K}, T_{r}=298 \mathrm{~K}$, $c=1000 \mathrm{Jkg}^{-1} \mathrm{~K}^{-1}, k=1 \mathrm{Wm}^{-1} \mathrm{~K}^{-1}, \alpha=1, \rho=2000 \mathrm{kgm}^{-3}, \chi_{1}=$ $30 \mathrm{Wm}^{-2} \mathrm{~K}^{-1}, \kappa=10^{3} \mathrm{~m}^{-1}$.

The appropriate values for physical constants are: $R=8.31431 \mathrm{JK}^{-1} \mathrm{~mol}^{-1}$, $\sigma=5.67 \times 10^{-8} \mathrm{Wm}^{-2} \mathrm{~K}^{4}$. 


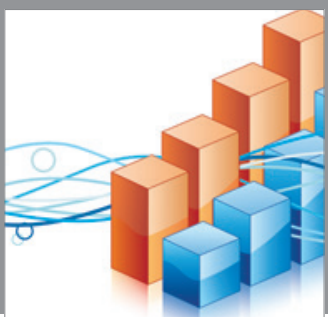

Advances in

Operations Research

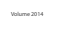

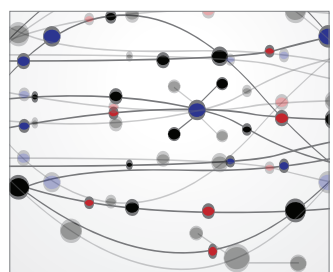

\section{The Scientific} World Journal
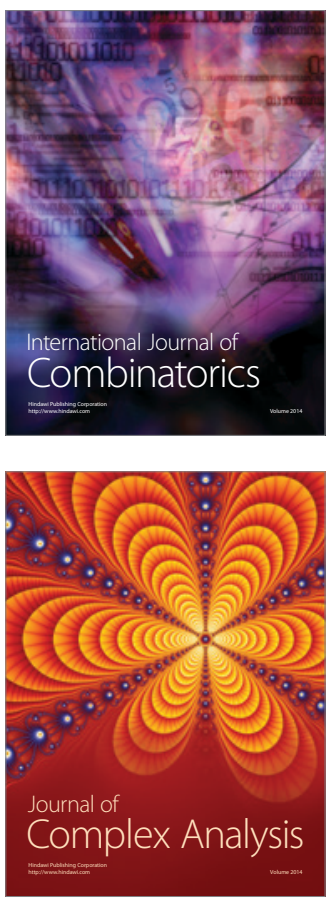

International Journal of

Mathematics and

Mathematical

Sciences
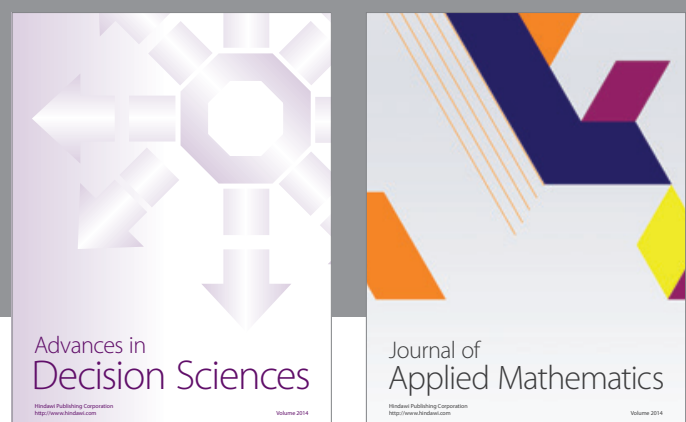

Journal of

Applied Mathematics
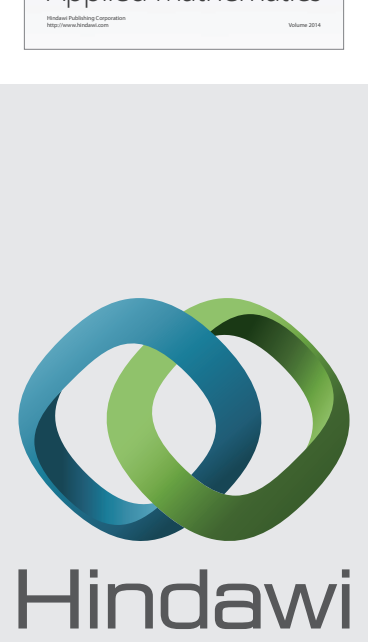

Submit your manuscripts at http://www.hindawi.com
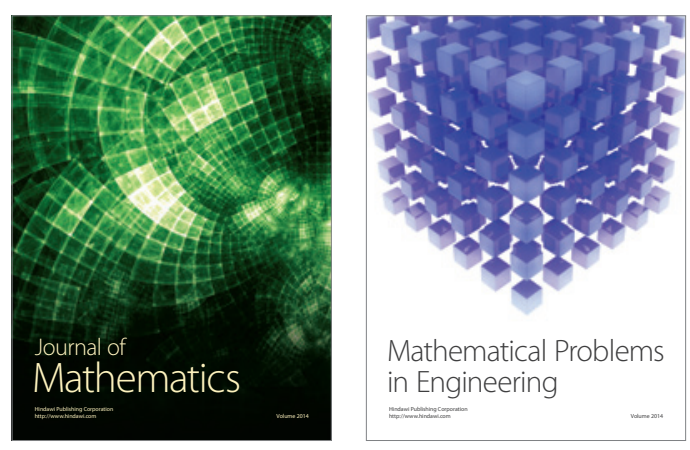

Mathematical Problems in Engineering
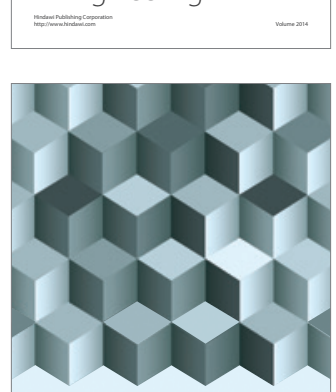

Journal of

Function Spaces
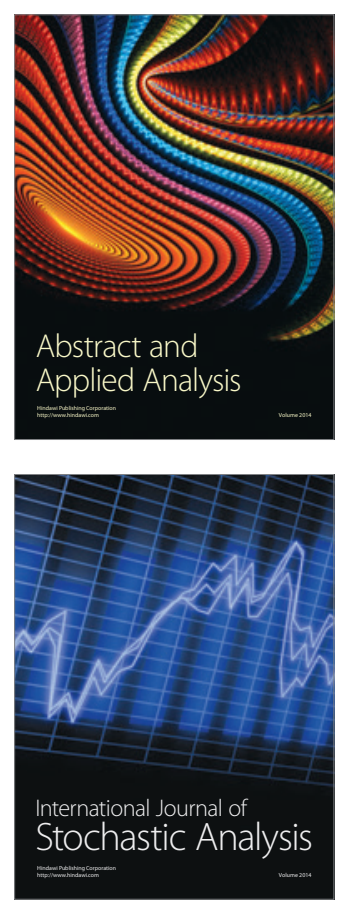

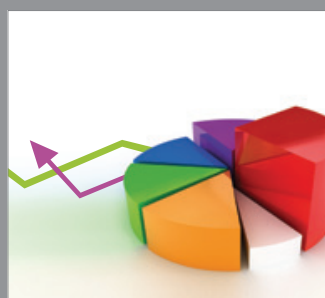

ournal of

Probability and Statistics

Promensencen
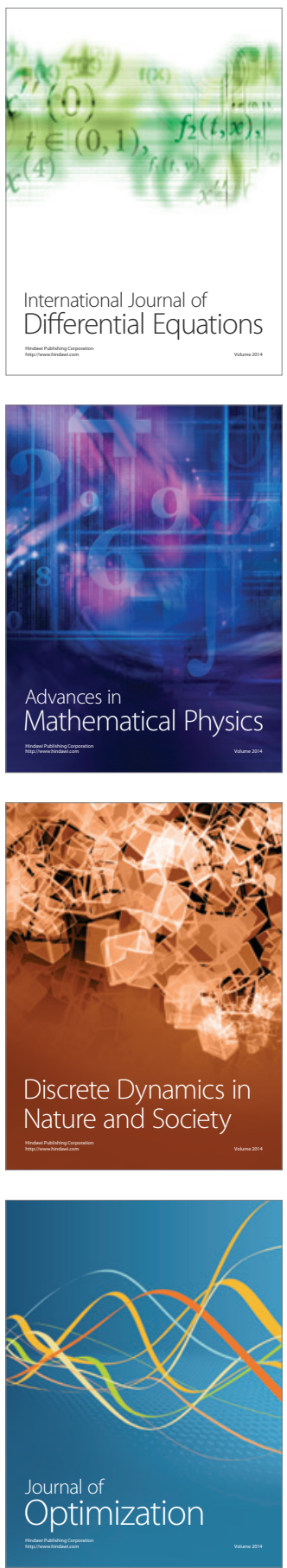